Article

\title{
On One Interpolation Type Fractional Boundary-Value Problem
}

\author{
Kateryna Marynets + (iD) \\ Delft Institute of Applied Mathematics, Delft University of Technology, 2628 XE Delft, The Netherlands; \\ K.Marynets@tudelft.nl \\ † Current address: Van Mourik Broekmanweg 6, 2628 XE Delft, The Netherlands.
}

Received: 11 December 2019; Accepted: 20 January 2020; Published: 28 January 2020

check for updates

\begin{abstract}
We present some new results on the approximation of solutions of a special type of fractional boundary-value problem. The focus of our research is a system of three fractional differential equations of the mixed order, subjected to the so-called "interpolation" type boundary restrictions. Under certain conditions, the aforementioned problem is simplified via a proper parametrization technique, and with the help of the numerical-analytic method, the approximate solutions are constructed.
\end{abstract}

Keywords: fractional differential system; interpolation type boundary condition; approximation of solution; parametrization technique

\section{Introduction}

Differential equations of the fractional order have a wide spectrum of applications, since they are often used to model problems in fluid dynamics, finance, biology, physics, engineering, etc. However, most of the exact methods of solution of the considered problems (see [1-11]) in the nonlinear case of differential equations and their systems are not applicable.

During the last few years, a number of papers devoted to the numerical-analytic methods of approximation of solutions of the fractional ordinary and partial differential equations were published. In particular, those were the well known homotopy perturbation method [12-15], Adomian's decomposition method [16-21], the variation iteration method [20,22,23], and the exp-function method [24].

Recently, in the series of papers (see discussions [25-28]), a completely new approach for study of a class of fractional periodic and anti-periodic boundary-value problems was developed. To construct the approximate solutions of the studied problems, the numerical-analytic method, based on the successive iterations, was used. This technique was originally suggested and successfully applied to the boundary-value problems for ordinary differential systems with strong nonlinearities not only in the equations, but also in the boundary conditions (see [29-32]).

This paper is a continuation of the obtained results in the aforementioned direction, and gives the important results for the study of a new class of the interpolation type fractional boundary-value problems. A suitable parametrization technique, sometimes called in literature a "freezing technique," transforms the given three-point boundary constraints into the two-point ones and allows us to construct the approximate solutions of the modified problem in an analytic form.

In Sections 2 and 3 the main terminology that will be used throughout the paper is given, and some important results already obtained in this direction are presented.

Sections 4-7 are devoted to the main result of the paper, particularly to the fractional boundary-value problems under the interpolation type boundary constraints. The primary interest of such boundary conditions is that we cannot apply the modification of the numerical-analytic scheme 
(see Section 3) straightforwardly. Therefore, we need to use a special "freezing," or parametrization technique to cope with these kinds of issues. Moreover, we reduce the order of the boundary conditions (from the initially three-point restrictions to the two-point ones) that plays an important role in the computational process. As a result: these techniques might be further studied in the more complicated cases of boundary conditions (e.g., two- and multipoint nonlinear boundary constraints), widely appearing in the mathematical models of applied sciences.

\section{Main Notations and Supplementary Statements}

Let $J=[a, b](-\infty<a<b<\infty)$ be a final interval of $\mathbb{R}$.

Definition 1. Function $\Gamma(z)$, defined by the integral

$$
\Gamma(z)=\int_{0}^{\infty} e^{-t} t^{z-1} d t
$$

which converges in the right half of the complex plane $\operatorname{Re}(z)>0$, is called the Gamma function.

Definition 2. [8] The left and right Riemann-Liouville fractional integrals of order $\alpha \in \mathbb{R}^{+}$are defined by

$$
{ }_{a} D_{t}^{-\alpha} f(t)=\frac{1}{\Gamma(\alpha)} \int_{a}^{t}(t-s)^{\alpha-1} f(s) d s, t>a, \alpha>0
$$

and

$$
{ }_{t} D_{b}^{-\alpha} f(t)=\frac{1}{\Gamma(\alpha)} \int_{t}^{b}(s-t)^{\alpha-1} f(s) d s, t<b, \alpha>0
$$

respectively, provided the right-hand sides are pointwise defined on $[a, b]$.

Definition 3. [8] The left and right Riemann-Liouville fractional derivatives of order $\alpha \in \mathbb{R}_{+}$are defined by

$$
{ }_{a} D_{t}^{\alpha} f(t)=\frac{d^{n}}{d t^{n}} D_{t}^{-(n-\alpha)} f(t)=\frac{1}{\Gamma(n-\alpha)} \frac{d^{n}}{d t^{n}} \int_{a}^{t}(t-s)^{n-\alpha-1} f(s) d s, t>a
$$

and

$$
{ }_{t} D_{b}^{\alpha} f(t)=(-1)^{n} \frac{d^{n}}{d t^{n}}{ }_{t} D_{b}^{-(n-\alpha)} f(t)=\frac{1}{\Gamma(n-\alpha)}(-1)^{n} \frac{d^{n}}{d t^{n}} \int_{t}^{b}(s-t)^{n-\alpha-1} f(s) d s, t<b
$$

respectively, where $n=[\alpha]+1,[\alpha]$ means the integer part of $\alpha$.

Definition 4. [8] The left and right Caputo fractional derivatives of order $\alpha \in \mathbb{R}_{+}$are defined by

$$
{ }_{a}^{C} D_{t}^{\alpha} f(t)={ }_{a} D_{t}^{\alpha}\left[f(t)-\sum_{k=0}^{n-1} \frac{f^{(k)}(a)}{k !}(t-a)^{k}\right]
$$

and

$$
{ }_{t}^{C} D_{b}^{\alpha} f(t)={ }_{t} D_{b}^{\alpha}\left[f(t)-\sum_{k=0}^{n-1} \frac{f^{(k)}(b)}{k !}(b-t)^{k}\right]
$$

respectively, where $n=[\alpha]+1$, for $\alpha \notin \mathbb{N}_{0} ; n=\alpha$ for $\alpha \in \mathbb{N}_{0}$.

In particular, when $0<\alpha<1$, then

$$
{ }_{a}^{C} D_{t}^{\alpha} f(t)={ }_{a} D_{t}^{\alpha}(f(t)-f(a))
$$

and

$$
{ }_{t}^{C} D_{b}^{\alpha} f(t)={ }_{t} D_{b}^{\alpha}(f(t)-f(b)) .
$$


Let us also formulate some subsidiary lemmas, first proven by Fečkan, Marynets in [25] and reformulated here over an interval $[a, b]$.

Lemma 1. Let $f(t)$ be a continuous function for $t \in[a, b]$. Then, for all $t \in[a, b]$, the following estimate is true:

$$
\begin{gathered}
\left|\frac{1}{\Gamma(j)} \int_{a}^{t}(t-\tau)^{j-1} f(\tau) d \tau-\left(\frac{t-a}{b-a}\right)^{j} \frac{1}{\Gamma(j)} \int_{a}^{b}(b-\tau)^{j-1} f(\tau) d \tau\right| \\
\leq \alpha_{1}^{j}(t) \max _{t \in[a, b]}|f(t)|, j \in(0,1],
\end{gathered}
$$

where

$$
\alpha_{1}^{j}(t)=\frac{2(b-t)^{j}}{\Gamma(j+1)}\left(1-\frac{b-t}{b-a}\right)^{j}
$$

Lemma 2. Let $\left\{\alpha_{m}^{j}(t)\right\}_{m \in \mathbb{N}}$ be a sequence of continuous functions at the interval $[a, b]$ given by

$$
\begin{aligned}
& \alpha_{m}^{j}(t):=\frac{1}{\Gamma(j)}\left[\int_{a}^{t}\left[(t-\tau)^{j-1}-\left(\frac{t-a}{b-a}\right)^{j}(b-\tau)^{j-1}\right] \alpha_{m-1}^{j}(\tau) d \tau\right. \\
&\left.+\left(\frac{t-a}{b-a}\right)^{j} \int_{t}^{b}(b-\tau)^{j-1} \alpha_{m-1}(\tau) d \tau\right], m \in \mathbb{N}, j \in(0,1],
\end{aligned}
$$

where $\alpha_{0}(t)=1$. Note $\alpha_{1}^{j}(t)$ is defined by Formula (2). Then, the following estimate holds:

$$
\alpha_{m+1}^{j}(t) \leq \frac{(b-a)^{m j}}{2^{m(2 j-1)} \Gamma^{m}(j+1)} \alpha_{1}(t) \leq \frac{(b-a)^{(m+1) j}}{2^{(m+1)(2 j-1)} \Gamma^{m+1}(j+1)},
$$

for $m \in \mathbb{N}_{0}$.

\section{Resent Results in the Study of the Periodic and Anti-Periodic Fractional Boundary Value Problems}

Since the approach of the numerical-analytic method [32] was appied to the fractional differential systems for the first time in [25-28], it is resonable to give an overview of the results that will allow the reader to follow and will open up possible perspectives for future research in this direction.

\subsection{Integral Representation of the Solution of the PFBVP}

To give an understanding of the numerical-analytic technique used for approximation of solutions of the fractional periodic and anti-periodic boundary-value problems (BVPs), let us first present the basic ideas of the aforementioned method.

Consider the simplest case of a periodic fractional BVP (PFBVP) (see discussion in [25]) of the form:

$$
\begin{array}{r}
{ }_{0}^{c} D_{t}^{p} x(t)=f(t, x(t)), p \in(0,1), x, f \in \mathbb{R}^{n}, \\
x(0)=x(T),
\end{array}
$$

where ${ }_{0}^{c} D_{t}^{p}$ is the generalized Caputo fractional derivative with the lower limit at 0 (see ([11], Definition 1.8; [33], Definition 2.3)), $t \in[0, T], x:[0, T] \rightarrow D, f: G \rightarrow \mathbb{R}^{n}$ are continuous functions, $G:=[0, T] \times D$, and $D \subset \mathbb{R}^{n}$ is a closed and bounded domain.

Together with the fractional differential system (FDS) (5) we consider an initial value problem for a perturbed system of the form:

$$
\begin{array}{r}
{ }_{0}^{c} D_{t}^{p} x(t)=f(t, x(t))+\Delta, \\
x(0)=x_{0},
\end{array}
$$


where $\Delta$ is a term to be defined.

Remark 1. Note, that the main advantage of the Caputo's approach is that the initial conditions for fractional differential equations with Caputo derivatives take on the same form as for the integer-order differential equations; i.e., contain the limit values of the integer-order derivatives of unknown functions at the lower limit $t=a$ (see [8]).

Using an integral representation of the Cauchy problem for the FDS with the initial data (8) we get:

$$
\begin{gathered}
x(t):=x_{0}+\frac{1}{\Gamma(p)} \int_{0}^{t}(t-s)^{p-1} f(s, x(s)+\Delta) d s \\
=x_{0}+\frac{1}{\Gamma(p)} \int_{0}^{t}(t-s)^{p-1} f(s, x(s)) d s+\frac{\Delta t^{p}}{\Gamma(p+1)}
\end{gathered}
$$

where $\Gamma(\cdot)$ is the Gamma function.

Substituting in (9) values $t=0, t=T$ and using the periodic boundary condition (6), we find an expression for the perturbation term:

$$
\Delta:=-\frac{p}{T^{p}} \int_{0}^{T}(T-s)^{p-1} f(s, x(s)) d s .
$$

Note that in order to apply the numerical-analytic technique for approximation of solutions of the problem (5) and (6) we need the following hypothesis to be held (see also discussions in [29-31]):

(H1) Function $f$ in the system (5) is bounded by a constant vector $M=\operatorname{col}\left(M_{1}, M_{2}, \ldots, M_{n}\right) \in$ $\mathbb{R}^{n}$ and it satisfies the Lipschitz condition with a non-negative real matrix $K=\left(k_{i j}\right)_{i, j=1}^{n}$; i.e., the following inequalities

$$
\begin{gathered}
|f(t, x)| \leq M, \\
|f(t, u)-f(t, v)| \leq K|u-v|
\end{gathered}
$$

are true for any $t \in[0, T], x, u, v \in D$.

(H2) The set

$$
D_{\beta}=\left\{x_{0} \in D:\left\{u \in \mathbb{R}^{n}:\left|u-x_{0}\right| \leq \beta\right\} \subset D\right\},
$$

is non-empty, where $\beta=\frac{M T^{p}}{2^{2 p-1} \Gamma(p+1)}$.

(H3) The spectral radius $r(Q)$ of matrix

$$
Q:=\frac{K T^{p}}{2^{2 p-1} \Gamma(p+1)}
$$

satisfies the estimate:

$$
r(Q)<1 .
$$

Then, the approximate solution of the PFBVP may be found from the iterative formula:

$$
\begin{gathered}
x_{m}\left(t, x_{0}\right):=x_{0}+\frac{1}{\Gamma(p)}\left[\int_{0}^{t}(t-s)^{p-1} f\left(s, x_{m-1}\left(s, x_{0}\right)\right) d s-\right. \\
\left.-\left(\frac{t}{T}\right)^{p} \int_{0}^{T}(T-s)^{p-1} f\left(s, x_{m-1}\left(s, x_{0}\right)\right) d s\right], m \in \mathbb{N},
\end{gathered}
$$

where $t \in[0, T], x_{0} \in D_{\beta}$ and $x_{0}\left(t, x_{0}\right)=x_{0}$ is considered as a zero approximation.

Note that the numerical values of the unknown vector $x_{0}$ are solutions of the so-called "bifurcation" system of the form:

$$
\Delta\left(x_{0}\right)=0,
$$

where $\Delta: D \rightarrow \mathbb{R}^{n}$ is defined by expression (10). 
Let us now pass to the more general cases of FDS.

\subsection{Study of the Lower Order Fractional Differential Systems}

In [26] we considered the FDS

$$
\begin{aligned}
& { }_{0}^{c} D_{t}^{p} x=f(t, x(t), y(t)), \\
& { }_{0}^{c} D_{t}^{q} y=g(t, x(t), y(t)),
\end{aligned}
$$

where first $n_{1}$ equations had the fractional order $p \in(0,1]$ and the rest $n_{2}$ equations were of the order $q \in(0,1]$.

Here $f: G_{f} \rightarrow \mathbb{R}^{n_{1}}, g: G_{g} \rightarrow \mathbb{R}^{n_{2}}$-are continuous functions; $G_{f}:=[0, T] \times D_{f}, G_{g}:=[0, T] \times D_{g}$, and $D_{f} \subset \mathbb{R}^{n_{1}}, D_{g} \subset \mathbb{R}^{n_{2}}$-are closed and bounded domains.

We were looking for a continuously differentiable solution of the system (11), subjected to the periodic boundary restrictions:

$$
x(0)=x(T), \quad y(0)=y(T) .
$$

Remark 2. The FDS (11) has a particular application to economic systems, since it may formulate a dynamical macroeconomic model of two national economies.

As mentioned in Section 3.1, particular requirements had to be met in order to apply the successive approximation's approach.

Indeed, in [26] we derived these conditions; i.e., we supposed that the BVP (11), (12) is such that the following conditions hold:

$\left(\mathbf{A}_{1}\right)$ Functions $f, g$ in the system (11) satisfy inequalities

$$
|f(t, x, y)| \leq M_{f}, \quad|g(t, x, y)| \leq M_{g}
$$

for any $t \in[0, T], x_{1}, x_{2} \in D_{f}, y_{1}, y_{2} \in D_{g}$ and some non-negative vectors $M_{f} \in \mathbb{R}^{n_{1}}, M_{g} \in \mathbb{R}^{n_{2}}$;

$\left.\mathbf{( B}_{1}\right)$ Functions $f$, $g$ satisfy the Lipschitz type conditions

$$
\begin{aligned}
& \left|f\left(t, x_{1}, y_{1}\right)-f\left(t, x_{2}, y_{2}\right)\right| \leq K_{11}\left|x_{1}-x_{2}\right|+K_{12}\left|y_{1}-y_{2}\right|, \\
& \left|g\left(t, x_{1}, y_{1}\right)-g\left(t, x_{2}, y_{2}\right)\right| \leq K_{21}\left|x_{1}-x_{2}\right|+K_{22}\left|y_{1}-y_{2}\right|,
\end{aligned}
$$

for any $t \in[0, T], x_{1}, x_{2} \in D_{f}, y_{1}, y_{2} \in D_{g}$ and some non-negative matrixes $K_{i j}, i, j \in\{1,2\}$;

$\left(\mathrm{C}_{1}\right)$ The sets

$$
\begin{gathered}
D_{\beta_{f}}:=\left\{x_{0} \in D_{f}:\left\{u \in \mathbb{R}^{n}:\left|u-x_{0}\right| \leq \beta_{f}\right\} \subset D_{f}\right\}, \\
D_{\beta_{g}}:=\left\{y_{0} \in D_{g}:\left\{v \in \mathbb{R}^{m}:\left|v-y_{0}\right| \leq \beta_{g}\right\} \subset D_{g}\right\}
\end{gathered}
$$

are non-empty, where

$$
\beta_{f}:=\frac{M_{f} T^{p}}{2^{2 p-1} \Gamma(p+1)}, \quad \beta_{g}:=\frac{M_{g} T^{q}}{2^{2 q-1} \Gamma(q+1)} ;
$$

$\left(\mathbf{D}_{1}\right)$ The spectral radius $r(Q)$ of the matrix $Q:=K \Gamma_{p q}$ satisfies an inequality $r(Q)<1$, where

$$
\Gamma_{p q}:=\max \left\{\frac{T^{p}}{2^{2 p-1} \Gamma(p+1)}, \frac{T^{q}}{2^{2 q-1} \Gamma(q+1)}\right\} .
$$


Then, we connected with the BVP (11), (12) the sequences of functions $\left\{x_{m}, y_{m}\right\}_{m \in \mathbb{N}}$, given by the iterative formulas:

$$
\begin{gathered}
x_{m}\left(t, x_{0}, y_{0}\right):=x_{0}+\frac{1}{\Gamma(p)}\left[\int_{0}^{t}(t-s)^{p-1} f\left(s, x_{m-1}\left(s, x_{0}, y_{0}\right), y_{m-1}\left(s, x_{0}, y_{0}\right)\right) d s\right. \\
\left.-\left(\frac{t}{T}\right)^{p} \int_{0}^{T}(T-s)^{p-1} f\left(s, x_{m-1}\left(s, x_{0}, y_{0}\right), y_{m-1}\left(s, x_{0}, y_{0}\right)\right) d s\right], \\
y_{m}\left(t, x_{0}, y_{0}\right):=y_{0}+\frac{1}{\Gamma(q)}\left[\int_{0}^{t}(t-s)^{q-1} g\left(s, x_{m-1}\left(s, x_{0}, y_{0}\right), y_{m-1}\left(s, x_{0}, y_{0}\right)\right) d s\right. \\
\left.-\left(\frac{t}{T}\right)^{q} \int_{0}^{T}(T-s)^{q-1} g\left(s, x_{m-1}\left(s, x_{0}, y_{0}\right), y_{m-1}\left(s, x_{0}, y_{0}\right)\right) d s\right], \\
x_{0}\left(t, x_{0}, y_{0}\right)=x_{0}, y_{0}\left(t, x_{0}, y_{0}\right)=y_{0},
\end{gathered}
$$

where $t \in[0, T], x_{0} \in D_{\beta_{f}}, y_{0} \in D_{\beta_{g}}$, coupled with the system of 'bifurcation' equations

$$
\begin{aligned}
& { }^{p} \Delta\left(x_{0}, y_{0}\right):=-\frac{p}{T^{p}} \int_{0}^{T}(T-s)^{p-1} f\left(s, x_{\infty}\left(s, x_{0}, y_{0}\right), y_{\infty}\left(s, x_{0}, y_{0}\right)\right) d s=0, \\
& { }^{q} \Delta\left(x_{0}, y_{0}\right):=-\frac{q}{T^{q}} \int_{0}^{T}(T-s)^{q-1} g\left(s, x_{\infty}\left(s, x_{0}, y_{0}\right), y_{\infty}\left(s, x_{0}, y_{0}\right)\right) d s=0 .
\end{aligned}
$$

In addition, the following theorems were proved.

Theorem 1. [26] Assume that conditions $\left(A_{1}\right)-\left(D_{1}\right)$ for the PFBPV (11), (12) hold. Then, for all fixed $x_{0} \in D_{\beta_{f}}$ $y_{0} \in D_{\beta_{g}}$ it holds that:

1. Functions of the sequence (13) are continuous and satisfy periodic boundary conditions

$$
x_{m}\left(0, x_{0}, y_{0}\right)=x_{m}\left(T, x_{0}, y_{0}\right), y_{m}\left(0, x_{0}, y_{0}\right)=y_{m}\left(T, x_{0}, y_{0}\right) .
$$

2. The sequences of functions (13) for $t \in[0, T]$ converge uniformly as $m \rightarrow \infty$ to the appropriate limit functions

$$
\begin{aligned}
& x_{\infty}\left(t, x_{0}, y_{0}\right)=\lim _{m \rightarrow \infty} x_{m}\left(t, x_{0}, y_{0}\right), \\
& y_{\infty}\left(t, x_{0}, y_{0}\right)=\lim _{m \rightarrow \infty} y_{m}\left(t, x_{0}, y_{0}\right) .
\end{aligned}
$$

3. The limit functions $x_{\infty}, y_{\infty}$ satisfy periodic boundary conditions

$$
x_{\infty}\left(0, x_{0}, y_{0}\right)=x_{\infty}\left(T, x_{0}, y_{0}\right), y_{\infty}\left(0, x_{0}, y_{0}\right)=y_{\infty}\left(T, x_{0}, y_{0}\right)
$$

and are the unique continuous solutions of the integral equations

$$
\begin{aligned}
x(t) & :=x_{0}+\frac{1}{\Gamma(p)}\left[\int_{0}^{t}(t-s)^{p-1} f(s, x(s), y(s)) d s\right. \\
- & \left.\left(\frac{t}{T}\right)^{p} \int_{0}^{T}(T-s)^{p-1} f(s, x(s), y(s)) d s\right], \\
y(t) & :=y_{0}+\frac{1}{\Gamma(q)}\left[\int_{0}^{t}(t-s)^{q-1} g(s, x(s), y(s)) d s\right. \\
- & \left.\left(\frac{t}{T}\right)^{q} \int_{0}^{T}(T-s)^{q-1} g(s, x(s), y(s)) d s\right] ;
\end{aligned}
$$


i.e., they are the unique solutions on $[0, T]$ of the Cauchy problem:

$$
\begin{array}{ll}
{ }_{0}^{c} D_{t}^{p} x=f(t, x(t), y(t))+{ }^{p} \Delta\left(x_{0}, y_{0}\right), & x(0)=x_{0}, \\
{ }_{0}^{c} D_{t}^{q} y=g(t, x(t), y(t))+{ }^{q} \Delta\left(x_{0}, y_{0}\right), & y(0)=y_{0},
\end{array}
$$

where

$$
\begin{aligned}
& { }^{p} \Delta\left(x_{0}, y_{0}\right):=-\frac{p}{T^{p}} \int_{0}^{T}(T-s)^{p-1} f\left(s, x_{\infty}\left(s, x_{0}, y_{0}\right), y_{\infty}\left(s, x_{0}, y_{0}\right)\right) d s, \\
& { }^{q} \Delta\left(x_{0}, y_{0}\right):=-\frac{q}{T^{q}} \int_{0}^{T}(T-s)^{q-1} g\left(s, x_{\infty}\left(s, x_{0}, y_{0}\right), y_{\infty}\left(s, x_{0}, y_{0}\right)\right) d s .
\end{aligned}
$$

4. The following error estimations hold:

$$
\left(\begin{array}{c}
\left|x_{\infty}\left(t, x_{0}, y_{0}\right)-x_{m}\left(t, x_{0}, y_{0}\right)\right| \\
\left|y_{\infty}\left(t, x_{0}, y_{0}\right)-y_{m}\left(t, x_{0}, y_{0}\right)\right|
\end{array}\right) \leq \Gamma_{p q} Q^{m}\left(I_{n}-Q\right)^{-1}\left(\begin{array}{c}
M_{f} \\
M_{g}
\end{array}\right),
$$

where $I_{n}$ is the $n$-dimensional identity matrix.

Theorem 2. [26] Let $x_{0} \in D_{\beta_{f}}, y_{0} \in D_{\beta_{g}}$ and $\zeta \in \mathbb{R}^{n_{1}}, \chi \in \mathbb{R}^{n_{2}}$ be some given vectors. Suppose that all conditions of Theorem 1 hold for the system of mixed FDEs (11). Then, the solutions $x=x\left(\cdot, x_{0}, y_{0}, \zeta\right)$, $y=y\left(\cdot, x_{0}, y_{0}, \chi\right)$ of the initial-value problem

$$
\begin{array}{ll}
{ }_{0}^{c} D_{t}^{p} x=f(t, x(t), y(t))+\zeta, & x(0)=x_{0}, \\
{ }_{0}^{c} D_{t}^{q} x=g(t, x(t), y(t))+\chi, & y(0)=y_{0},
\end{array}
$$

where $t \in[0, T], \zeta \in \mathbb{R}^{n_{1}}, \chi \in \mathbb{R}^{n_{2}}$ are the control parameters and $x_{0} \in D_{\beta_{f}}, y_{0} \in D_{\beta_{g}}$, satisfy also boundary conditions (12), if and only if

$$
\zeta:=^{p} \Delta\left(x_{0}, y_{0}\right), \quad \chi:={ }^{q} \Delta\left(x_{0}, y_{0}\right),
$$

where ${ }^{p} \Delta,{ }^{q} \Delta$ are given by the left hand-side of the equation (15). In that case

$$
x\left(t, x_{0}, y_{0}, \zeta\right)=x_{\infty}\left(t, x_{0}, y_{0}\right), \quad y\left(t, x_{0}, y_{0}, \chi\right)=y_{\infty}\left(t, x_{0}, y_{0}\right) .
$$

The next corollary was the main result of the study, giving us conditions of the connection between the pair of limit functions $\left(x^{*}\left(\cdot, x_{0}, y_{0}\right), y^{*}\left(\cdot, x_{0}, y_{0}\right)\right)$ of the sequences (13) and the exact solution of the mixed type FBVP (11), (12).

Corollary 1. [26] Let conditions $\left(A_{1}\right)-\left(D_{1}\right)$ hold. Then, $x_{\infty}\left(\cdot, x_{0}^{*}, y_{0}^{*}\right), y_{\infty}\left(\cdot, x_{0}^{*}, y_{0}^{*}\right)$ are the solutions of $(11)$, (12), if and only if a pair $\left(x_{0}^{*}, y_{0}^{*}\right)$ is the solution of the determining system:

$$
\Delta\left(x_{0}, y_{0}\right):=\left({ }^{p} \Delta\left(x_{0}, y_{0}\right),{ }^{q} \Delta\left(x_{0}, y_{0}\right)\right)=0,
$$

where ${ }^{p} \Delta,{ }^{q} \Delta$ are given by relations (15).

\subsection{PFBVP with a Higher Order Caputo Type Fractional Derivative}

As a generalization of the aforementioned in Sections 3.1 and 3.2 problems, in [27] we studied the generalized fractional differential system

$$
{ }_{0}^{c} D_{t}^{p} x(t)=f(t, x(t)), p \in(m, m+1), m \in \mathbb{N}
$$

with periodic boundary conditions

$$
x^{(i)}(0)=x^{(i)}(T), i=\overline{0, m},
$$


where $t \in[0, T], T>0, x \in C^{m}([0, T], D), D \subset \mathbb{R}^{n}$ is open, $f \in C\left(G, \mathbb{R}^{n}\right), G:=[0, T] \times D$.

It was shown (see discussions in [27]) that if the FBVP (16), (17) satisfies the hypothesis:

$\left(\mathbf{A}_{2}\right)$ There is a non-negative vector $M=\operatorname{col}\left(M_{1}, M_{2}, \ldots, M_{n}\right) \in \mathbb{R}^{n}, M_{i} \geq 0$ and a non-negative real matrix $L=\left(l_{i j}\right)_{i, j=1}^{n}, l_{i, j} \geq 0$ such that

$$
\begin{gathered}
|f(t, x)| \leq M, \forall(t, x) \in G, \\
|f(t, u)-f(t, v)| \leq L|u-v|, \forall t \in[0, T], \forall\{u, v\} \subset D ;
\end{gathered}
$$

$\left(\mathbf{B}_{2}\right)$ The open set

$$
D_{\beta}=\left\{\tilde{\xi}_{0} \in D:\left\{u \in \mathbb{R}^{n}:\left|u-\xi_{0}\right| \leq \beta\right\} \subset D\right\}
$$

is non-empty for $\beta:=T^{p} \kappa_{m} M$, where

$$
\kappa_{m}=2\left(\sum_{j=1}^{m} \frac{1}{j ! \Gamma(p-j+2)} \max _{z \in[0,1]}\left|B_{j}(z)-B_{j}\right|+\frac{1}{\Gamma(p+1)}\right)
$$

and $B_{i}$ and $B_{i}(z)$ are the Bernoulli numbers and polynomials;

$\left(C_{2}\right)$ The spectral radius $r(Q)$ of matrix $Q:=\kappa_{m} T^{p} L$ satisfies the estimate $r(Q)<1$, one can associate an iteration scheme

$$
\begin{gathered}
x_{k+1}(t)=\xi_{0}+\sum_{j=1}^{m} \frac{T^{j-1}}{j !}\left[B_{j}\left(\frac{t}{T}\right)-B_{j}\right]\left[-\frac{1}{\Gamma(p-j+1)} \int_{0}^{T}(T-s)^{p-j} f\left(s, x_{k}(s)\right) d s\right. \\
\left.+\frac{(p-m) T^{m-j+1}}{\Gamma(p-j+2)} \int_{0}^{T}(T-s)^{p-m-1} f\left(s, x_{k}(s)\right) d s\right] \\
+\frac{1}{\Gamma(p)} \int_{0}^{t}(t-s)^{p-1} f\left(s, x_{k}(s)\right) d s-\frac{(p-m) t^{p}}{T^{p-m} \Gamma(p+1)} \int_{0}^{T}(T-s)^{p-m-1} f\left(s, x_{k}(s)\right) d s, \\
k=0,1, \ldots,
\end{gathered}
$$

where $t \in[0, T], x_{0}(t)=\xi_{0} \in D_{\beta}$, with the solution of the given BVP (16), (17).

Similarly to Theorem 1 in Section 3.2 it was proved that a sequence of functions (18) is convergent to the limit function, which is a solution of the given BVP, if the corresponding determining equation

$$
\Delta=-\frac{p-m}{T^{p-m}} \int_{0}^{T}(T-s)^{p-m-1} f(s, x(s)) d s=0
$$

has a root.

Moreover in the case of the asymptotically constant scalar problems for the fractional differential equations of the form

$$
{ }_{0}^{c} D_{t}^{p} x(t)=g(x(t))+h(t), p \in(m, m+1), m \in \mathbb{N}, t \in[0, T]
$$

with boundary conditions (17) for $n=1, g \in C(\mathbb{R}, \mathbb{R}), h \in C([0, T], \mathbb{R})$ under hypothesis $\left(A_{2}\right)-\left(C_{2}\right)$ and if

$\left(\mathbf{D}_{2}\right)$ There are $g_{ \pm} \in \mathbb{R}$ such that

$$
\lim _{x \rightarrow \pm \infty} g(x)=g_{ \pm}
$$

conditions of existence and nonexistence of solutions are obtained. 


\subsection{Anti-Periodic Fractional BVP}

Recently we considered a boundary-value problem for a FDS with anti-periodic boundary conditions of the form:

$$
\begin{array}{r}
{ }_{0}^{c} D_{t}^{p} x=f(t, x(t)), p \in(0,1), t \in[0, T], x, f \in \mathbb{R}^{n}, \\
x(0)=-x(T),
\end{array}
$$

where $x:[0, T] \rightarrow D, f: G \rightarrow \mathbb{R}^{n}$ are continuous functions, $G:=[0, T] \times D$ and $D \subset \mathbb{R}^{n}$ is a closed and bounded domain.

Under conditions (H1)-(H4) in Section 3.1 the approximate solution of the anti-periodic FBVP (AFBPV) (19), (20) was constructed in the form:

$$
\begin{gathered}
x_{m}\left(t, \xi_{0}\right):=\xi_{0}+\frac{1}{\Gamma(p)}\left[\int_{0}^{t}(t-s)^{p-1} f\left(s, x_{m-1}\left(s, \xi_{0}\right)\right) d s\right. \\
\left.-\left(\frac{t}{T}\right)^{p} \int_{0}^{T}(T-s)^{p-1} f\left(s, x_{m-1}\left(s, \xi_{0}\right)\right) d s\right]-2\left(\frac{t}{T}\right)^{p} \xi_{0}
\end{gathered}
$$

with a modified form of zero approximation, given by the relation:

$$
x_{0}\left(t, \xi_{0}\right)=\left(1-2\left(\frac{t}{T}\right)^{p}\right) \xi_{0} .
$$

Remark 3. A choice of the zero-approximation in the form (22) is optimal in the sense that it improves the estimate of difference between the m-th approximation $x_{m}\left(\cdot, \xi_{0}\right)$ and its limit function $x_{\infty}\left(\cdot, \xi_{0}\right)$. In the case of selection $x_{0}\left(t, \xi_{0}\right)=x_{0}$, as it was for the PFBVPs (5), (6) and (11), (12), it would lead to additional terms in the estimates that would increase the error of computations.

In addition to the aforementioned convergence conditions and corollaries about the connection of the limit function of the sequence (21) to the exact solution of the AFBVP (19), (20), a proper solvability analysis of the original problem was provided.

In particular, the following statements have been proven.

Theorem 3. [28] Let conditions of Theorem 1 in [28] hold and one can take some $m \geq 1$ and the set $\Omega \subset \mathbb{R}^{n}$ such that the relation

$$
\left|\Phi_{m}\right| \triangleright_{\partial \Omega} Q^{m}\left(I_{n}-Q\right)^{-1} M,
$$

holds, where $\partial \Omega$ is understood as a boundary of domain $\Omega$. Besides, if the Brouwer degree of the vector-field $\Phi_{m}$ over the set $\Omega$ with respect to zero satisfies an inequality

$$
\operatorname{deg}\left(\Phi_{m}, \Omega, 0\right) \neq 0
$$

then their exists a point $\xi_{0}^{*} \in \Omega$ such that

$$
x_{\infty}(t)=x_{\infty}\left(t, \xi_{0}^{*}\right)=\lim _{m \rightarrow \infty} x_{m}\left(t, \xi_{0}^{*}\right)
$$

is the solution of the anti-periodic BVP (19), (20) with the initial condition

$$
x^{*}(0)=\xi_{0}^{*} \in \Omega
$$


Theorem 4. [28] Let conditions of Theorem 1 in [28] be held, and there exists some number $m \in \mathbb{N}$ and a point $\bar{\xi}_{0} \in \Omega$, such that the component-wise inequality:

$$
\begin{array}{r}
\left|\Delta_{m}\left(\bar{\xi}_{0}\right)\right| \leq \sup _{\xi \in D}\left[K R+Q\left(I_{n}-Q\right)^{-1} R+\frac{2 \Gamma(p+1)}{T^{p}} I_{n}\right]\left|\xi_{0}-\bar{\xi}_{0}\right| \\
+Q^{m}\left(I_{n}-Q\right)^{-1} M
\end{array}
$$

is not true, where matrix $K$ and vector $M$ appear in (H1), matrix $Q$ is defined in (H2), and matrix $R:=$ $\sup _{t \in[0, T]}\left|1+2\left(\frac{t}{T}\right)^{p}\right| I_{n}$.

Then, there does not exist a point $\bar{\xi}_{0} \in \Omega$, for which the antiperiodic BVP (19), (20) has a solution $x=x(t)$ such that $x(0)=\xi_{0}^{*}$.

Remark 4. On the basis of Theorem 4, we established an algorithm of approximate localization of the point $\xi_{0}^{*}$, that defines solution $x(\cdot)$ of the original BVP (19), (20). The set $\Omega$ was rewritten as an association of the finite value of subsets :

$$
\Omega:=\bigcup_{i=1}^{N} \Omega_{i}
$$

In every subset $\Omega_{i}$ from (23) we picked a point

$$
\bar{\xi}_{0}^{i} \in \Omega_{i}
$$

and for some fixed $m$ calculated the $m$-th approximation $x_{m}\left(\cdot, \bar{\xi}_{0}^{i}\right)$, by using the recurrent Formula (21). Then, we obtained the value of the determining function

$$
\Delta_{m}\left(\xi_{0}\right):=-\frac{p}{T^{p}} \int_{0}^{T}(T-s)^{p-1} f\left(s, x_{m}\left(s, \xi_{0}\right)\right) d s-\frac{2 \Gamma(p+1)}{T^{p}} \xi_{0}
$$

and excluded from the set (23) subsets $\Omega_{i}$, for which the inequality is not true.

When $N$ and $m$ tend to $\infty, \Omega_{m, N}$ "follows" to the set $\Omega^{*}$ that may contain value $\xi_{0}^{*}$ and defines solution of the anti-periodic BVP (19), (20).

Every point $\tilde{\xi}_{0} \in \Omega_{m, N}$ one can observe as an approximation to the value $\xi_{0}^{*}$ that determines solution of the given BVP (19), (20). In this case it is clear that

$$
\left|\tilde{\xi_{0}}-\xi_{0}^{*}\right| \leq \sup _{\xi_{0} \in \Omega_{m, N}}\left|\tilde{\xi}_{0}-\xi_{0}\right|
$$

and the value of function $x_{m}\left(t, \tilde{\xi}_{0}\right)$ that is calculated by iterative Formula (21), may be taken as an approximate solution of the BVP (19), (20).

\section{Boundary-Value Problem for a Mixed FDS under the Interpolation Type Boundary Constraints}

Consider a system of FDEs

$$
\left\{\begin{array}{l}
{ }_{a}^{C} D_{t}^{p} x=f(t, x(t), y(t), z(t)), \\
{ }_{a}^{C} D_{t}^{q} y=g(t, x(t), y(t), z(t)), \\
{ }_{a}^{C} D_{t}^{r} z=h(t, x(t), y(t), z(t)),
\end{array}\right.
$$


for some $p, q, r \in(0,1]$, where $f: G_{f} \rightarrow \mathbb{R}, g: G_{g} \rightarrow \mathbb{R}$ and $h: G_{h} \rightarrow \mathbb{R}$ are continuous functions; $G_{f}:=[a, b] \times D_{f}, G_{g}:=[a, b] \times D_{g}, G_{h}:=[a, b] \times D_{h}$, and $D_{f} \subset \mathbb{R}, D_{g} \subset \mathbb{R}$ and $D_{h} \subset \mathbb{R}$ are closed and bounded domains; and the system is subjected to the interpolation type boundary constraints

$$
\begin{gathered}
x(a)=d_{1}, \\
y\left(t_{1}\right)=d_{2}, t_{1} \in(a, b), \\
z(b)=d_{3},
\end{gathered}
$$

where $d_{i} \in \mathbb{R}, i=\overline{1,3}$ are some given values.

The problem is to find a triple $(x(\cdot), y(\cdot), z(\cdot))$ that satisfies the mixed-order FDS (24) and the three-point boundary conditions of the interpolation type (25) in the class of continuously differentiable functions $x, y, z:[a, b] \rightarrow \mathbb{R}$.

\section{Parametrization Approach and the Numerical-Analytic Technique}

Let us define some components of solution of the fractional boundary-value problem (FBVP) at the points $t=a, t=b$ by parameters:

$$
\begin{aligned}
& y(a)=\xi_{2}, z(a)=\xi_{3}, \\
& x(b)=\lambda_{1}, y(b)=\lambda_{2},
\end{aligned}
$$

and rewrite the boundary conditions in the form

$$
\begin{gathered}
x(a)+x(b)=d_{1}+\lambda_{1}, \\
y(b)=\lambda_{2}, \\
z(b)=d_{3} ;
\end{gathered}
$$

i.e., instead of the original three-point restrictions (25), we study a problem with some parametrized two-point constraints (27).

Remark 5. Note, that under additional conditions (26) the parametrized FBVP (24), (27) is equivalent to the original BVP of the interpolation type (24), (25).

Now we construct the proper sequences of functions:

$$
\begin{gathered}
x_{m}(t, \xi, \lambda) \\
:=d_{1}+\frac{1}{\Gamma(p)} \int_{a}^{t}(t-s)^{p-1} f\left(s, x_{m-1}(s, \xi, \lambda), y_{m-1}(s, \xi, \lambda), z_{m-1}(s, \xi, \lambda)\right) d s \\
-\left(\frac{t-a}{b-a}\right)^{p} \frac{1}{\Gamma(p)} \int_{a}^{b}(b-s)^{p-1} f\left(s, x_{m-1}(s, \xi, \lambda), y_{m-1}(s, \xi, \lambda), z_{m-1}(s, \xi, \lambda)\right) d s \\
+\left(\frac{t-a}{b-a}\right)^{p}\left[\lambda_{1}-d_{1}\right], \\
\quad y_{m}(t, \xi, \lambda) \\
-\left(\frac{t-a}{b-a}\right)^{q} \frac{1}{\Gamma(q)} \int_{a}^{b}(b-s)^{q-1} g\left(s, x_{m-1}(s, \xi, \lambda), y_{m-1}(s, \xi, \lambda), z_{m-1}(s, \xi, \lambda)\right) d s \\
+\left(\frac{t-a}{b-a}\right)^{q}\left[\lambda_{2}-\xi_{2}\right],
\end{gathered}
$$




$$
\begin{gathered}
z_{m}(t, \xi, \lambda) \\
:=\xi_{3}+\frac{1}{\Gamma(r)} \int_{a}^{t}(t-s)^{r-1} h\left(s, x_{m-1}(s, \xi, \lambda), y_{m-1}(s, \xi, \lambda), z_{m-1}(s, \xi, \lambda)\right) d s \\
-\left(\frac{t-a}{b-a}\right)^{r} \frac{1}{\Gamma(r)} \int_{a}^{b}(b-s)^{r-1} h\left(s, x_{m-1}(s, \xi, \lambda), y_{m-1}(s, \xi, \lambda), z_{m-1}(s, \xi, \lambda)\right) d s \\
+\left(\frac{t-a}{b-a}\right)^{r}\left[d_{3}-\xi_{3}\right],
\end{gathered}
$$

$\forall m \in \mathbb{N}$ and $t \in[a, b], \xi=\operatorname{col}\left(\xi_{1}, \xi_{2}\right) \in \Xi, \lambda=\operatorname{col}\left(\lambda_{1}, \lambda_{2}\right) \in \Lambda$, in such a way that they satisfy the FDS (24) and the boundary restrictions (27) in advance.

Note, that the functions

$$
\begin{aligned}
& x_{0}(t, \xi, \lambda)=d_{1}+\left(\frac{t-a}{b-a}\right)^{p}\left[\lambda_{1}-d_{1}\right], \\
& y_{0}(t, \xi, \lambda)=\xi_{2}+\left(\frac{t-a}{b-a}\right)^{q}\left[\lambda_{2}-\xi_{2}\right],
\end{aligned}
$$

and

$$
z_{0}(t, \xi, \lambda)=\xi_{3}+\left(\frac{t-a}{b-a}\right)^{r}\left[d_{3}-\xi_{3}\right]
$$

are taken as functions of a zero-approximation.

Assume that the FBVP (24), (27) satisfies the following conditions:

(i) Functions $f, g, h$ in the system (24) are bounded by some non-negative constants $M_{f}, M_{g}, M_{h} \in \mathbb{R}$.

(ii) Functions $f, g, h$ in the system (24) satisfy the Lipschitz type condition

$$
\left(\begin{array}{c}
\left|f\left(t, x_{1}, y_{1}, z_{1}\right)-f\left(t, x_{2}, y_{2}, z_{2}\right)\right| \\
\left|g\left(t, x_{1}, y_{1}, z_{1}\right)-g\left(t, x_{2}, y_{2}, z_{2}\right)\right| \\
\left|h\left(t, x_{1}, y_{1}, z_{1}\right)-h\left(t, x_{2}, y_{2}, z_{2}\right)\right|
\end{array}\right) \leq K\left(\begin{array}{c}
\left|x_{1}-x_{2}\right| \\
\left|y_{1}-y_{2}\right| \\
\left|z_{1}-z_{2}\right|
\end{array}\right)
$$

for any $t \in[a, b], x_{1}, x_{2} \in D_{f}, y_{1}, y_{2} \in D_{g}, z_{1}, z_{2} \in D_{h}$ and some non-negative matrix $K=\left(k_{i j}\right)_{i, j=1}^{3}$. (iii) The sets

$$
\begin{aligned}
D_{\beta_{f}} & :=\left\{x_{0} \in D_{f}:\left\{u \in \mathbb{R}:\left|u-x_{0}\right| \leq \beta_{f}\right\} \subset D_{f}\right\}, \\
D_{\beta_{g}} & :=\left\{y_{0} \in D_{g}:\left\{v \in \mathbb{R}:\left|v-y_{0}\right| \leq \beta_{g}\right\} \subset D_{g}\right\}, \\
D_{\beta_{h}} & :=\left\{z_{0} \in D_{h}:\left\{w \in \mathbb{R}:\left|w-z_{0}\right| \leq \beta_{h}\right\} \subset D_{h}\right\}
\end{aligned}
$$

are non-empty, where

$$
\beta_{f}:=\frac{M_{f}(b-a)^{p}}{2^{2 p-1} \Gamma(p+1)}, \quad \beta_{g}:=\frac{M_{g}(b-a)^{q}}{2^{2 q-1} \Gamma(q+1)}, \quad \beta_{h}:=\frac{M_{h}(b-a)^{r}}{2^{2 r-1} \Gamma(r+1)} .
$$

The following convergence theorem holds.

Theorem 5. Let functions $f: G_{f} \rightarrow \mathbb{R}, g: G_{g} \rightarrow \mathbb{R}$ and $h: G_{h} \rightarrow \mathbb{R}$ satisfy conditions (i)-(iii) in the domains $D_{l}, l=\{f, g, h\}$ with a matrix $K$ for which

$$
\lambda_{Q}<1,
$$

where $\lambda_{Q}$ is the spectral radius of matrix

$$
Q=\Gamma_{p q r} K
$$


for $\Gamma_{p q r}:=\max \left\{\frac{(b-a)^{p}}{2^{2 p-1} \Gamma(p+1)}, \frac{(b-a)^{q}}{2^{2 q-1} \Gamma(q+1)}, \frac{(b-a)^{r}}{2^{2 r-1} \Gamma(r+1)}\right\}$.

Then, for all fixed $\xi \in \Xi$ and $\lambda \in \Lambda$ the statements to be hold:

1. All functions of the sequences (28)-(30) are absolutely continuous on the interval $[a, b]$, have values in the domains $D_{l}, l=\{f, g, h\}$ respectively, and satisfy the two-point boundary conditions

$$
\begin{gathered}
x_{m}(a, \xi, \lambda)+x_{m}(b, \xi, \lambda)=d_{1}+\lambda_{1}, \\
y_{m}(b, \xi, \lambda)=\lambda_{2}, \\
z_{m}(b, \xi, \lambda)=d_{3} .
\end{gathered}
$$

2. The sequences of functions (28)-(30) for $t \in[a, b]$ converge uniformly as $m \rightarrow \infty$ to the limit functions:

$$
\begin{aligned}
& x_{\infty}(t, \xi, \lambda)=\lim _{m \rightarrow \infty} x_{m}(t, \xi, \lambda), \\
& y_{\infty}(t, \xi, \lambda)=\lim _{m \rightarrow \infty} y_{m}(t, \xi, \lambda), \\
& z_{\infty}(t, \xi, \lambda)=\lim _{m \rightarrow \infty} z_{m}(t, \xi, \lambda) .
\end{aligned}
$$

3. The limit functions satisfy the initial conditions

$$
\begin{gathered}
x_{\infty}(a, \xi, \lambda)=d_{1}, \\
y_{\infty}(a, \xi, \lambda)=\xi_{2}, \\
z_{\infty}(a, \xi, \lambda)=\xi_{3}
\end{gathered}
$$

and the two-point boundary conditions

$$
\begin{gathered}
x_{\infty}(a, \xi, \lambda)+x_{m}(b, \xi, \lambda)=d_{1}+\lambda_{1}, \\
y_{\infty}(b, \xi, \lambda)=\lambda_{2}, \\
z_{\infty}(b, \xi, \lambda)=d_{3} .
\end{gathered}
$$

4. The functions $x_{\infty}(\cdot, \xi, \lambda), y_{\infty}(\cdot, \xi, \lambda), z_{\infty}(\cdot, \xi, \lambda)$ are the unique continuously differentiable solutions of the integral equations

$$
\begin{aligned}
& x(t):=d_{1}+\frac{1}{\Gamma(p)} \int_{a}^{t}(t-s)^{p-1} f(s, x(s), y(s), z(s)) d s \\
& -\left(\frac{t-a}{b-a}\right)^{p} \frac{1}{\Gamma(p)} \int_{a}^{T}(b-s)^{p-1} f(s, x(s), y(s), z(s)) d s \\
& +\left(\frac{t-a}{b-a}\right)^{p}\left[\lambda_{1}-d_{1}\right] \\
& y(t):=\xi_{2}+\frac{1}{\Gamma(q)} \int_{a}^{t}(t-s)^{q-1} g(s, x(s), y(s), z(s)) d s \\
& -\left(\frac{t-a}{b-a}\right)^{q} \frac{1}{\Gamma(q)} \int_{a}^{b}(b-s)^{q-1} g(s, x(s), y(s), z(s)) d s \\
& +\left(\frac{t-a}{b-a}\right)^{q}\left[\lambda_{2}-\xi_{2}\right] \\
& z(t):=\xi_{3}+\frac{1}{\Gamma(r)} \int_{a}^{t}(t-s)^{r-1} h(s, x(s), y(s), z(s)) d s \\
& -\left(\frac{t-a}{b-a}\right)^{r} \frac{1}{\Gamma(r)} \int_{a}^{b}(b-s)^{r-1} h(s, x(s), y(s), z(s)) d s \\
& +\left(\frac{t-a}{b-a}\right)^{r}\left[d_{3}-\xi_{3}\right] .
\end{aligned}
$$


In other words, $x_{\infty}(\cdot, \xi, \lambda), y_{\infty}(\cdot, \xi, \lambda), z_{\infty}(\cdot, \xi, \lambda)$ satisfy the Cauchy problem for the modified system of FDEs:

$$
\left\{\begin{array}{c}
{ }_{a}^{C} D_{t}^{p} x=f(t, x(t), y(t), z(t))+\frac{p}{(b-a)^{p}} \Delta_{1}, \\
{ }_{a}^{C} D_{t}^{q} y=g(t, x(t), y(t), z(t))+\frac{q}{(b-a)^{q}} \Delta_{2}, \\
{ }_{a}^{C} D_{t}^{r} z=h(t, x(t), y(t), z(t))+\frac{r}{(b-a)^{r}} \Delta_{3}, \\
x(a)=d_{1}, y(a)=\xi_{2}, z(a)=\xi_{3},
\end{array}\right.
$$

where $\Delta_{i}(\xi, \lambda): \Xi \times \Lambda \rightarrow \mathbb{R}, i=\overline{1,3}$ are the mappings given by formulas:

$$
\begin{aligned}
\Delta_{1}(\xi, \lambda) & :=-\int_{a}^{b}(b-s)^{p-1} f(s, x(s), y(s), z(s)) d s+\Gamma(p)\left[\lambda_{1}-d_{1}\right], \\
\Delta_{2}(\xi, \lambda) & :=-\int_{a}^{b}(b-s)^{q-1} g(s, x(s), y(s), z(s)) d s+\Gamma(q)\left[\lambda_{2}-\xi_{2}\right], \\
\Delta_{3}(\xi, \lambda) & :=-\int_{a}^{b}(b-s)^{r-1} h(s, x(s), y(s), z(s)) d s+\Gamma(r)\left[d_{3}-\xi_{3}\right] .
\end{aligned}
$$

5. The following error estimate holds:

$$
\left(\begin{array}{c}
\left|x_{\infty}(t, \xi, \lambda)-x_{m}(t, \xi, \lambda)\right| \\
\left|y_{\infty}(t, \xi, \lambda)-y_{m}(t, \xi, \lambda)\right| \\
\left|z_{\infty}(t, \xi, \lambda)-z_{m}(t, \xi, \lambda)\right|
\end{array}\right) \leq \Gamma_{p q r} Q^{m}\left(I_{3}-Q\right)^{-1} M,
$$

where $I_{3}$ is the identity three-dimensional matrix and $M=\operatorname{col}\left(M_{f}, M_{g}, M_{h}\right)$ is a vector, defined in the condition (i).

Proof. As already mentioned, the sequences of functions (28)-(30) are constructed in such a form that they satisfy the parametrized boundary restrictions in advance. Due to this fact, the statement 1 of the theorem holds.

Let us prove that all functions $x_{m}(\cdot, \xi, \lambda), y_{m}(\cdot, \xi, \lambda), z_{m}(\cdot, \xi, \lambda)$ of the sequences (28)-(30) remain in the domains $D_{l}, l=\{f, g, h\}$, where each of them is defined; i.e., the iteration process can be continued until infinity. For this purpose we estimate the differences:

$$
\begin{gathered}
\left|x_{m}(t, \xi, \lambda)-x_{0}(t, \xi, \lambda)\right| \\
=\mid \frac{1}{\Gamma(p)} \int_{a}^{t}(t-s)^{p-1} f\left(s, x_{m-1}(s, \xi, \lambda), y_{m-1}(s, \xi, \lambda), z_{m-1}(s, \xi, \lambda)\right) d s \\
-\left(\frac{t-a}{b-a}\right)^{p} \frac{1}{\Gamma(p)} \int_{a}^{b}(b-s)^{p-1} f\left(s, x_{m-1}(s, \xi, \lambda), y_{m-1}(s, \xi, \lambda), z_{m-1}(s, \xi, \lambda)\right) d s \mid \\
\leq \alpha_{1}^{p}(t) M_{f} \leq \frac{(b-a)^{p} M_{f}}{2^{2 p-1} \Gamma(p+1)}=\beta_{f}, \\
=\mid \frac{1}{\Gamma(q)} \int_{a}^{t}(t-s)^{q-1} g\left(s, x_{m-1}(s, \xi, \lambda), y_{m-1}(s, \xi, \lambda), z_{m-1}(s, \xi, \lambda)\right) d s \\
-\left(\frac{t-a}{b-a}\right)^{q} \frac{1}{\Gamma(q)} \int_{a}^{b}(b-s)^{q-1} g\left(s, x_{m-1}(s, \xi, \lambda), y_{m-1}(s, \xi, \lambda), z_{m-1}(s, \xi, \lambda)\right) d s \mid \\
\leq \alpha_{1}^{q}(t) M_{g} \leq \frac{(b-a)^{q} M_{g}}{2^{2 q-1} \Gamma(q+1)}=\beta_{g},
\end{gathered}
$$




$$
\begin{gathered}
\left|z_{m}(t, \xi, \lambda)-z_{0}(t, \xi, \lambda)\right| \\
=\mid \frac{1}{\Gamma(r)} \int_{a}^{t}(t-s)^{r-1} h\left(s, x_{m-1}(s, \xi, \lambda), y_{m-1}(s, \xi, \lambda), z_{m-1}(s, \xi, \lambda)\right) d s \\
-\left(\frac{t-a}{b-a}\right)^{r} \frac{1}{\Gamma(h)} \int_{a}^{b}(b-s)^{r-1} h\left(s, x_{m-1}(s, \xi, \lambda), y_{m-1}(s, \xi, \lambda), z_{m-1}(s, \xi, \lambda)\right) d s \mid \\
\leq \alpha_{1}^{r}(t) M_{h} \leq \frac{(b-a)^{r} M_{h}}{2^{2 r-1} \Gamma(r+1)}=\beta_{h},
\end{gathered}
$$

for all $m \in \mathbb{N}, t \in[a, b], \xi \in \Xi, \lambda \in \Lambda$.

Let us introduce a vector

$$
M_{m}:=\left(\begin{array}{c}
X_{m} \\
Y_{m} \\
Z_{m}
\end{array}\right)=K^{m-1}\left(\begin{array}{c}
M_{f} \\
M_{g} \\
M_{h}
\end{array}\right),
$$

where $K$ is the matrix from the Lipschitz condition (ii), and the sequences of functions

$$
\begin{aligned}
\mu_{m}(t):=\frac{1}{\Gamma(p)} \max \left\{\int_{a}^{t}\left[(t-\tau)^{p-1}-\left(\frac{t-a}{b-a}\right)^{p}(b-\tau)^{p-1}\right] \mu_{m-1}(\tau) d \tau\right. \\
+\left(\frac{t-a}{b-a}\right)^{p} \int_{t}^{b}(b-\tau)^{p-1} \mu_{m-1}(\tau) d \tau, \\
\int_{a}^{t}\left[(t-\tau)^{p-1}-\left(\frac{t-a}{b-a}\right)^{p}(b-\tau)^{p-1}\right] \eta_{m-1}(\tau) d \tau \\
\quad+\left(\frac{t-a}{b-a}\right)^{p} \int_{t}^{b}(b-\tau)^{p-1} \eta_{m-1}(\tau) d \tau, \\
\int_{a}^{t}\left[(t-\tau)^{p-1}-\left(\frac{t-a}{b-a}\right)^{p}(b-\tau)^{p-1}\right] v_{m-1}(\tau) d \tau \\
\left.+\left(\frac{t-a}{b-a}\right)^{p} \int_{t}^{b}(b-\tau)^{p-1} v_{m-1}(\tau) d \tau\right\}, \\
\eta_{m}(t):=\frac{1}{\Gamma(q)} \max \left\{\int_{a}^{t}\left[(t-\tau)^{q-1}-\left(\frac{t-a}{b-a}\right)^{q}(b-\tau)^{q-1}\right] \mu_{m-1}(\tau) d \tau\right. \\
\quad+\left(\frac{t-a}{b-a}\right)^{q} \int_{t}^{b}(b-\tau)^{q-1} \mu_{m-1}(\tau) d \tau, \\
\int_{a}^{t}\left[(t-\tau)^{q-1}-\left(\frac{t-a}{b-a}\right)^{q}(b-\tau)^{q-1}\right] \eta_{m-1}(\tau) d \tau \\
\quad+\left(\frac{t-a}{b-a}\right)^{q} \int_{t}^{b}(b-\tau)^{q-1} \eta_{m-1}(\tau) d \tau, \\
\int_{a}^{t}\left[(t-\tau)^{q-1}-\left(\frac{t-a}{b-a}\right)^{q}(b-\tau)^{q-1}\right] v_{m-1}(\tau) d \tau \\
\left.+\left(\frac{t-a}{b-a}\right)^{q} \int_{t}^{b}(b-\tau)^{q-1} v_{m-1}(\tau) d \tau\right\},
\end{aligned}
$$




$$
\begin{aligned}
v_{m}(t):=\frac{1}{\Gamma(r)} \max \left\{\int_{a}^{t}\left[(t-\tau)^{r-1}-\left(\frac{t-a}{b-a}\right)^{r}(b-\tau)^{r-1}\right] \mu_{m-1}(\tau) d \tau\right. \\
+\left(\frac{t-a}{b-a}\right)^{r} \int_{t}^{b}(b-\tau)^{r-1} \mu_{m-1}(\tau) d \tau, \\
\int_{a}^{t}\left[(t-\tau)^{r-1}-\left(\frac{t-a}{b-a}\right)^{r}(b-\tau)^{r-1}\right] \eta_{m-1}(\tau) d \tau \\
\quad+\left(\frac{t-a}{b-a}\right)^{r} \int_{t}^{b}(b-\tau)^{r-1} \eta_{m-1}(\tau) d \tau, \\
\int_{a}^{t}\left[(t-\tau)^{r-1}-\left(\frac{t-a}{b-a}\right)^{r}(b-\tau)^{r-1}\right] v_{m-1}(\tau) d \tau \\
\left.+\left(\frac{t-a}{b-a}\right)^{r} \int_{t}^{b}(b-\tau)^{r-1} v_{m-1}(\tau) d \tau\right\},
\end{aligned}
$$

for $m \geq 2$ with $\mu_{1}(t)=\alpha_{1}^{p}(t), \eta_{1}(t)=\alpha_{1}^{q}(t)$ and $v_{1}(t)=\alpha_{1}^{r}(t)$. Note that $M_{m+1}=K M_{m}$.

Suppose that the estimates hold:

$$
\begin{aligned}
\left|x_{m}(t, \xi, \lambda)-x_{m-1}(t, \xi, \lambda)\right| & \leq X_{m} \mu_{m}(t), \\
\left|y_{m}(t, \xi, \lambda)-y_{m-1}(t, \xi, \lambda)\right| & \leq Y_{m} \eta_{m}(t), \\
\left|z_{m}(t, \xi, \lambda)-z_{m-1}(t, \xi, \lambda)\right| & \leq Z_{m} v_{m}(t),
\end{aligned}
$$

for all $t \in[a, b], \xi \in \Xi, \lambda \in \Lambda$, and $m \in \mathbb{N}$. Indeed, the inequalities (56) are satisfied for $m=1$ due to the estimates (50)-(52). Using the mathematical induction method, we derive

$$
\begin{gathered}
\left|x_{m+1}(t, \xi, \lambda)-x_{m}(t, \xi, \lambda)\right| \\
\leq \frac{k_{11}}{\Gamma(p)}\left[\int_{a}^{t}\left((t-s)^{p-1}-\left(\frac{t-a}{b-a}\right)^{p}(b-s)^{p-1}\right)\left|x_{m}(s, \xi, \lambda)-x_{m-1}(s, \xi, \lambda)\right| d s\right. \\
\left.\quad+\left(\frac{t-a}{b-a}\right)^{p} \int_{t}^{b}(b-s)^{p-1}\left|x_{m}(s, \xi, \lambda)-x_{m-1}(s, \xi, \lambda)\right| d s\right] \\
+\frac{k_{12}}{\Gamma(p)}\left[\int_{a}^{t}\left((t-s)^{p-1}-\left(\frac{t-a}{b-a}\right)^{p}(b-s)^{p-1}\right)\left|y_{m}(s, \xi, \lambda)-y_{m-1}(s, \xi, \lambda)\right| d s\right. \\
+\frac{k_{13}}{\Gamma(p)}\left[\int_{a}^{t}\left((t-s)^{p-1}-\left(\frac{t-a}{b-a}\right)^{p}(b-s)^{p-1}\right)\left|z_{m}(s, \xi, \lambda)-z_{m-1}(s, \xi, \lambda)\right| d s\right. \\
\left.\quad+\left(\frac{t-a}{b-a}\right)^{p} \int_{t}^{b}(b-s)^{p-1}\left|z_{m}(s, \xi, \lambda)-z_{m-1}(s, \xi, \lambda)\right| d s\right] \\
\leq \frac{k_{11} X_{m}\left[\int_{a}^{t}\left((t-s)^{p-1}-\left(\frac{t-a}{\Gamma(p)}\right)^{p}(b-s)^{p-1}\right) \mu_{m}(s) d s\right.}{\left.+\left(\frac{t-a}{b-a}\right)^{p} \int_{t}^{b}(b-s)^{p-1} \mu_{m}(s) d s\right]}
\end{gathered}
$$




$$
\begin{gathered}
+\frac{k_{12}}{\Gamma(p)} Y_{m}\left[\int_{a}^{t}\left((t-s)^{p-1}-\left(\frac{t-a}{b-a}\right)^{p}(b-s)^{p-1}\right) \eta_{m}(s) d s\right. \\
\left.+\left(\frac{t-a}{b-a}\right)^{p} \int_{t}^{b}(b-s)^{p-1} \eta_{m}(s) d s\right] \\
+\frac{k_{13}}{\Gamma(p)} Z_{m}\left[\int_{a}^{t}\left((t-s)^{p-1}-\left(\frac{t-a}{b-a}\right)^{p}(b-s)^{p-1}\right) v_{m}(s) d s\right. \\
\left.+\left(\frac{t-a}{b-a}\right)^{p} \int_{t}^{b}(b-s)^{p-1} v_{m}(s) d s\right] \\
\leq\left(k_{11} X_{m}+k_{12} Y_{m}+k_{13} Z_{m}\right) \mu_{m+1}(t)=X_{m+1} \mu_{m+1}(t),
\end{gathered}
$$

for all $t \in[a, b], \xi \in \Xi, \lambda \in \Lambda$. This proves the first inequality of (56). The last two estimates in (56) are proven analogically.

Let us define a function

$$
\alpha_{1}(\cdot)=\max \left\{\alpha_{1}^{p}(\cdot), \alpha_{1}^{q}(\cdot), \alpha_{1}^{r}(\cdot)\right\},
$$

satisfying an inequality:

$$
\alpha_{1}(t) \leq \Gamma_{p q r} .
$$

Taking into account relations (53)-(55), we can derive the following estimates:

$$
\begin{aligned}
\mu_{m}(t) \leq \Gamma_{p q r}^{m-1 p} \alpha_{1}(t) & \leq \Gamma_{p q r}^{m} \quad \eta_{m}(t) \leq \Gamma_{p q r}^{m-1 q} \alpha_{1}(t) \leq \Gamma_{p q r}^{m}, \\
v_{m}(t) & \leq \Gamma_{p q r}^{m-1 r} \alpha_{1}(t) \leq \Gamma_{p q r}^{m} .
\end{aligned}
$$

Then, the vector relation

$$
\left(\begin{array}{c}
\left|x_{m+1}(t, \xi, \eta)-x_{m}(t, \xi, \eta)\right| \\
\left|y_{m+1}(t, \xi, \eta)-y_{m}(t, x \xi, \eta)\right| \\
\left|z_{m+1}(t, \xi, \eta)-z_{m}(t, \xi, \eta)\right|
\end{array}\right) \leq \Gamma_{p q r}^{m+1} M_{m+1}=\Gamma_{p q r} Q^{m}\left(\begin{array}{c}
M_{f} \\
M_{g} \\
M_{h}
\end{array}\right)
$$

holds, which implies

$$
\begin{gathered}
\left(\begin{array}{l}
\left|x_{m+j}(t, \xi, \eta)-x_{m}(t, \xi, \eta)\right| \\
\left|y_{m+j}(t, \xi, \eta)-y_{m}(t, \xi, \eta)\right| \\
\left|z_{m+j}(t, \xi, \eta)-z_{m}(t, \xi, \eta)\right|
\end{array}\right) \\
\leq\left(\begin{array}{c}
\sum_{i=1}^{j}\left|x_{m+i}(t, \xi, \eta)-x_{m+i-1}(t, \xi, \eta)\right| \\
\sum_{i=1}^{j}\left|y_{m+i}(t, \xi, \eta)-y_{m+i-1}(t, \xi, \eta)\right| \\
\sum_{i=1}^{j}\left|z_{m+i}(t, \xi, \eta)-z_{m+i-1}(t, \xi, \eta)\right|
\end{array}\right) \leq \Gamma_{p q r} Q^{m} \sum_{i=0}^{j-1} Q^{i}\left(\begin{array}{c}
M_{f} \\
M_{g} \\
M_{h}
\end{array}\right) .
\end{gathered}
$$

Since for the spectral radius of matrix $Q$ an inequality (33) holds, we conclude that

$$
\sum_{i=0}^{j-1} Q^{i} \leq\left(I_{3}-Q\right)^{-1}, \quad \lim _{m \rightarrow \infty} Q^{m}=O_{3}
$$

where $O_{3}$ is the three-dimensional zero matrix. By letting $j \rightarrow \infty$ in the relation (57), we derive the estimate (49). This means that the sequences of functions $\left\{x_{m}(\cdot, \xi, \lambda)\right\},\left\{y_{m}(\cdot, \xi, \lambda)\right\},\left\{z_{m}(\cdot, \xi, \lambda)\right\}$, given by formulas (28)-(30) accordingly, converge uniformly in the domains $[a, b] \times \Xi \times \Lambda$ to the corresponding limit functions $x_{\infty}(\cdot, \xi, \lambda), y_{\infty}(\cdot, \xi, \lambda)$, and $z_{\infty}(\cdot, \xi, \lambda)$. As a result, it is obvious that functions $x_{\infty}(\cdot, \xi, \lambda), y_{\infty}(\cdot, \xi, \lambda)$, and $z_{\infty}(\cdot, \xi, \lambda)$ solve the integral equations (41)-(43). 
The uniqueness of the solutions can be proven in a similar way as in [26] by generalization of the interval to $[a, b]$ and consideration of three functions $x(\cdot), y(\cdot), z(\cdot)$, instead of two, as it was done in the aforementioned paper.

\section{Main Result}

Let us study the Cauchy problem for a perturbed fractional differential system

$$
\left\{\begin{array}{l}
{ }_{a}^{C} D_{t}^{p} x=f(t, x(t), y(t), z(t))+\frac{p}{(b-a)^{p}} \zeta_{1}, \\
{ }_{a}^{C} D_{t}^{q} y=g(t, x(t), y(t), z(t))+\frac{q}{(b-a)^{q}} \zeta_{2}, \\
{ }_{a}^{C} D_{t}^{r} z=h(t, x(t), y(t), z(t))+\frac{r}{(b-a)^{r}} \zeta_{3},
\end{array}\right.
$$

with the given initial data (45), where $t \in[a, b]$ and $\zeta=\operatorname{col}\left(\zeta_{1}, \zeta_{2}, \zeta_{3}\right) \in \mathbb{R}^{3}$-the constant perturbation term.

Theorem 6. Let $\xi \in \Xi, \lambda \in \Lambda$, and $\zeta \in \mathbb{R}^{3}$ be some given vectors. Suppose that all conditions of Theorem 5 hold for the system of FDEs (24). Then, the solutions $x=x\left(\cdot, \xi, \lambda, \zeta_{1}\right), y=y\left(\cdot, \xi, \lambda, \zeta_{2}\right), z=z\left(\cdot, \xi, \lambda, \zeta_{3}\right)$ of the initial-value problem (58), (45) satisfy the boundary conditions (25), if and only if

$$
\begin{aligned}
& \zeta_{1}:=\Delta_{1}(\xi, \lambda), \\
& \zeta_{2}:=\Delta_{2}(\xi, \lambda), \\
& \zeta_{3}:=\Delta_{3}(\xi, \lambda),
\end{aligned}
$$

where $\Delta_{i}, i=\overline{1,3}$ are given by formulas (46)-(48).

In that case

$$
\begin{aligned}
& x\left(t, \xi, \lambda, \zeta_{1}\right)=x_{\infty}(t, \xi, \lambda), \\
& y\left(t, \xi, \lambda, \zeta_{2}\right)=y_{\infty}(t, \xi, \lambda), \\
& z\left(t, \xi, \lambda, \zeta_{3}\right)=z_{\infty}(t, \xi, \lambda) .
\end{aligned}
$$

Theorem 7. Let conditions of Theorem 5 hold. Then $x_{\infty}\left(\cdot, \xi^{*}, \lambda^{*}\right), y_{\infty}\left(\cdot, \xi^{*}, \lambda^{*}\right), z_{\infty}\left(\cdot, \xi^{*}, \lambda^{*}\right)$ are the solutions of the FBVP (24), (25) if and only if the point $\left(\xi^{*}, \lambda^{*}\right)$ is the solution of the determining system:

$$
\begin{array}{r}
\Delta_{i}(\xi, \lambda)=0, i=\overline{1,3}, \\
y_{\infty}\left(t_{1}, \xi, \lambda\right)=d_{2} .
\end{array}
$$

Proof. The result follows directly from Theorem 6 by observing that the perturbed FDS (44) coincides with equation (24) if and only if the point $\left(\xi^{*}, \lambda^{*}\right)$ satisfies the system of determining Equations (61) and (62).

\section{Some Solvability and Applicability Remarks}

In addition to the results above, we can prove that the system of determining Equations (61) and (62) determines all possible solutions of the interpolation type FBVP (24), (25).

Theorem 8. Assume that conditions of Theorem 5 hold. If there exists a point $(\xi, \lambda) \in \Xi \times \Lambda$ that satisfies the system of determining Equations (61), (62), then the FBVP (24), (25) has a solution $\left(x^{0}(\cdot), y^{0}(\cdot), z^{0}(\cdot)\right)$ such that

$$
y^{0}(a)=\xi_{2}^{0}, z^{0}(a)=\xi_{3}^{0}, x^{0}(b)=\lambda_{1}^{0}, y^{0}(b)=\lambda_{2}^{0},
$$


and this solution is given by the limit functions of the sequences (28)-(30):

$$
\begin{aligned}
& x^{0}(t)=x_{\infty}\left(t, \xi^{0}, \lambda^{0}\right)=\lim _{m \rightarrow \infty} x_{m}\left(t, \xi^{0}, \lambda^{0}\right), t \in[a, b], \\
& y^{0}(t)=y_{\infty}\left(t, \xi^{0}, \lambda^{0}\right)=\lim _{m \rightarrow \infty} y_{m}\left(t, \xi^{0}, \lambda^{0}\right), t \in[a, b], \\
& z^{0}(t)=z_{\infty}\left(t, \xi^{0}, \lambda^{0}\right)=\lim _{m \rightarrow \infty} z_{m}\left(t, \xi^{0}, \lambda^{0}\right), t \in[a, b] .
\end{aligned}
$$

Conversely, if the FBVP (24), (25) has a solution $\left(x^{0}(\cdot), y^{0}(\cdot), z^{0}(\cdot)\right)$, then $\left(x^{0}(\cdot), y^{0}(\cdot), z^{0}(\cdot)\right)$ necessarily has the form of (63)-(65) and the system of determining Equations (61), (62) is satisfied with

$$
\xi_{2}^{0}=y^{0}(a), \xi_{3}^{0}=z^{0}(a), \lambda_{1}^{0}=x^{0}(b), \lambda_{2}^{0}=y^{0}(b) .
$$

Proof. If values $\xi_{2}^{0}, \xi_{3}^{0}, \lambda_{1}^{0}, \lambda_{2}^{0}$ which satisfy the system of determining Equations (61), (62) exist, then according to Theorem 5, the functions (63)-(65) are the solutions of the given FBVP (24), (25).

On the other hand, if $\left(x^{0}(\cdot), y^{0}(\cdot), z^{0}(\cdot)\right)$ are the solutions of the original problem (24), (25), then these functions are the solutions of the Cauchy problem (58), (45) with

$$
\zeta_{1}=0, \zeta_{2}=0, \zeta_{3}=0 \text { and } y(a)=\xi_{2}, z(a)=\xi_{3} .
$$

As $\left(x^{0}(\cdot), y^{0}(\cdot), z^{0}(\cdot)\right)$ satisfy the linear two-point parametrized boundary restrictions (27), by virtue of relations (60) of Theorem 6 the equalities (63)-(65) hold.

Moreover,

$$
\zeta_{1}=0, \zeta_{2}=0, \zeta_{3}=0,
$$

where $\xi_{2}^{0}, \xi_{3}^{0}, \lambda_{1}^{0}, \lambda_{2}^{0}$ are defined by (66). From (68) we have that the determining system (61), (62) is satisfied, if $\xi, \lambda$ are given by (66).

We have thus specified in $(66)$ the values of a pair $(\xi, \lambda)$ that satisfy the system of determining Equations (61), (62), which proves the theorem.

Remark 6. In practice it is more suitable to consider the approximate determining equations

$$
\begin{gathered}
\Delta_{i, m}(\xi, \lambda)=0, i=\overline{1,3}, \\
y_{m}\left(t_{1}, \xi, \lambda\right)=d_{2},
\end{gathered}
$$

instead of the exact ones (61), (62), where $\Delta_{i, m}: \Xi \times \Lambda \rightarrow \mathbb{R}, i=\overline{1,3}$ is the $m$-th determining function, given by formulas

$$
\begin{array}{r}
\Delta_{1, m}(\xi, \lambda):=-\int_{a}^{b}(b-s)^{p-1} f\left(s, x_{m}(s, \xi, \lambda), y_{m}(s, \xi, \lambda), z_{m}(s, \xi, \lambda)\right) d s \\
+\Gamma(p)\left[\lambda_{1}-d_{1}\right], \\
\Delta_{2, m}(\xi, \lambda):=-\int_{a}^{b}(b-s)^{p-1} g\left(s, x_{m}(s, \xi, \lambda), y_{m}(s, \xi, \lambda), z_{m}(s, \xi, \lambda)\right) d s \\
+\Gamma(q)\left[\lambda_{2}-\xi_{2}\right], \\
\Delta_{3, m}(\xi, \lambda):=-\int_{a}^{b}(b-s)^{r-1} h\left(s, x_{m}(s, \xi, \lambda), y_{m}(s, \xi, \lambda), z_{m}(s, \xi, \lambda)\right) d s \\
+\Gamma(r)\left[d_{3}-\xi_{3}\right],
\end{array}
$$

and $x_{m}(\cdot, \xi, \lambda), y_{m}(\cdot, \xi, \lambda), z_{m}(\cdot, \xi, \lambda)$ are defined by the iterative formulas (28)-(30) respectively. 
Funding: No funding to be declared.

Acknowledgments: The author is thankful to the reviewers for their notes and comments that helped to improve the paper.

Conflicts of Interest: There is no conflict of interest.

\section{References}

1. Cattani, C. Harmonic Wavelet Solutions of the Schrodinger Equation. Int. J. Fluid Mech. Res. 2003, 30, 463-472. [CrossRef]

2. Al-Ghafri, K.S.; Rezazadeh, H. Solitons and other solutions of (3+1)-dimensional space-time fractional modified KdV-Zakharov-Kuznetsov equation. Appl. Math. Nonlinear Sci. 2019, 4, 289-304. [CrossRef]

3. Gao, W.; Ghanbari, B.; Baskonus, H.M. New numerical simulations for some real world problems with Atangana-Baleanu fractional derivative. Chaos Solitons Fractals 2019, 128, 34-43. [CrossRef]

4. Gao, W.; Yel, G.; Baskonus, H.M.; Cattani, C. Complex solitons in the conformable $(2+1)$-dimensional Ablowitz-Kaup-Newell-Segur equation. Aims Math. 2020, 5, 507-521. [CrossRef]

5. Gao, W.; Ismael, H.F.; Mohammed, S.A.; Baskonus, H.M.; Bulut, H. Complex and real optical soliton properties of the paraxial non-linear Schrödinger equation in kerr media with M-fractional. Front. Phys. 2019. [CrossRef]

6. Kilbas, A.A.A.; Srivastava, H.M.; Trujillo, J.J. Theory and Applications of Fractional Differential Equations; Elsevier: Amsterdam, The Netherlands, 2006.

7. Khan, M.A.; Hammouch, Z.; Baleanu, D. Modeling the dynamics of hepatitis E via the Caputo-Fabrizio derivative. Math. Model. Nat. Phenom. 2019, 14, 311. [CrossRef]

8. Podlubny, I. Fractional Differential Equations; Academic Press: Cambridge, MA, USA, 1999.

9. Yokus, A.; Gülbahar, S. Numerical solutions with linearization techniques of the fractional Harry Dym equation. Appl. Math. Nonlinear Sci. 2019, 4, 35-42. [CrossRef]

10. Youssef1, I.K.; El Dewaik, M.H. Solving Poisson's equations with fractional order using haarwavelet. Appl. Math. Nonlinear Sci. 2017, 2, 271-284. [CrossRef]

11. Zhou, Y. Basic Theory of Fractional Differential Equations; World Scientific: Singapore, 2014.

12. Gao, W.; Veeresha, P.; Prakasha, D.G.; Baskonus, H.M.; Yel, G. A powerful approach for fractional Drinfeld-Sokolov-Wilson equation with Mittag-Leffler law. Alex. Eng. J. 2019. [CrossRef]

13. Momani, S.; Odibat, Z. Homotopy perturbation method for nonlinear partial differential equations of fractional order. Phys. Lett. A 2007, 365, 345-350. [CrossRef]

14. Odibat, Z.; Momani, S. Modified homotopy perturbation method: application to quadratic Riccati differential equation of fractional order. Chaos Solitons Fractals 2008, 36, 167-174. [CrossRef]

15. Zhang, Y.; Cattani, C.; Yang, J. Local fractional homotopy perturbation method for solving non-homogeneous heat conduction equations in fractal domains. Entropy 2015, 17, 6753-6764. [CrossRef]

16. Daftardar-Gejji, V.; Jafari, H. Adomian decomposition: A tool for solving a system of fractional differential equations. J. Math. Anal. Appl. 2005, 301, 508-518. [CrossRef]

17. Jafari, H.; Gejji, V.D. Solving a system of nonlinear fractional differential equations using Adomain decomposition. Appl. Math. Comput. 2006 196, 644-651. [CrossRef]

18. Lensic, D. The decomposition method for initial value problems. Appl. Math. Comput. 2006, 181, $206-213$.

19. Lensic, D. The decomposition method for Cauchy advection-diffusion problems. Appl. Math. Comput. 2005, 49, 525-537.

20. Momani, S.; Odibat, Z. Numerical comparison of methods for solving linear differential equations of fractional order. Chaos Solitons Fractals 2007, 31, 1248-1255. [CrossRef]

21. Momani, S.; Al-Khaled, K. Numerical solutions for systems of fractional differential equations by the decomposition method. Appl. Math. Comput. 2005, 162, 1351-1365. [CrossRef]

22. Jafari, H.; Tajadodi, H. He's Variational Iteration Method for Solving Fractional Riccati Differential Equation. Int. J. Differ. Equ. 2010, 2010. [CrossRef]

23. Odibat, Z.; Momani, S. Application of variational iteration method to nonlinear differential equations of fractional order. Int. J. Nonlinear Sci. Numer. Simul. 2006, 1, 15-27. [CrossRef]

24. Guner, O.; Bekir, A. The Exp-function method for solving nonlinear space-time fractional differential equations in mathematical physics. J. Assoc. Arab. Univ. Basic Appl. Sci. 2017, 24, 277-282. [CrossRef] 
25. Fečkan, M.; Marynets, K. Approximation approach to periodic BVP for fractional differential systems. Eur. Phys. J. Spec. Top. 2017, 226, 3681-3692. [CrossRef]

26. Fečkan, M.; Marynets, K. Approximation approach to periodic BVP for mixed fractional differential systems. J. Comput. Appl. Math. 2018, 339, 208-217. [CrossRef]

27. Fečkan, M.; Marynets, K.; Wang, J.R. Periodic boundary value problems for higher order fractional differential systems. Math. Methods Appl. Sci. 2019, 42, 3616-3632. [CrossRef]

28. Marynets, K. Solvability analysis of a special type fractional differential system. Comput. Appl. Math. 2019, 39, 3. [CrossRef]

29. Marynets, K. On construction of the approximate solution of the special type integral boundary-value problem. Electron. J. Qual. Theory Differ. Equ. 2016, 2016, 1-14. [CrossRef]

30. Ronto, M.; Varha, Y.; Marynets, K. Further results on the investigation of solutions of integral boundary value problems. Tatra Mt. Publ. 2015, 63, 247-267. [CrossRef]

31. Ronto, M.I.; Marynets', K.V. On the parametrization of boundary-value problems with two-point nonlinear boundary conditions. Nonlinear Oscil. 2012, 14, 379-413. [CrossRef]

32. Ronto, M.; Samoilenko, A.M. Numerical-Analytic Methods in the Theory of Boundary-Value Problems; World Scientific: Singapore, 2000.

33. Wang, J.R.; Fečkan, M.; Zhou, Y. A survey on impulsive fractional differential equations. Fract. Calc. Appl. Anal. 2016, 19, 806-831. [CrossRef]

(C) 2020 by the authors. Licensee MDPI, Basel, Switzerland. This article is an open access article distributed under the terms and conditions of the Creative Commons Attribution (CC BY) license (http:/ / creativecommons.org/licenses/by/4.0/). 